ORIENTAL JOURNAL OF
ISSN: 0974-6471
June 2017,
COMPUTER SCIENCE \& TECHNOLOGY

\title{
A Novel Approach to address Sensor Interoperability Using Gabor Filter
}

\author{
NEHA BHATIA ${ }^{1}$, HIMANI ${ }^{2}$ and CHANDER KANT ${ }^{3}$ \\ 1,3Department of Computer science and Application, Kurukshetra University, Kurukshetra, India \\ ${ }^{2}$ Doon Valley Institute of Engineering and Technology, Karnal, India
}

http://dx.doi.org/10.13005/ojcst/10.02.27

(Received: May 05, 2017; Accepted: May 13, 2017)

\begin{abstract}
Biometric authentication using fingerprint is one of the unique and reliable method of verification processes. Biometric System suffers a signiûcant loss of performance when the sensor is changed during enrollment and authentication process. In this paper fingerprint sensor interoperability problem is addressed using Gabor ûlter and classifying images into good and poor quality. Gabor ûlters play an important role in many application areas for the enhancement of various types of fingerprint images. Gabor ûlters can remove noise, preserve the real ridges and valley structures, and it is used for fingerprint image enhancement. Experimental results on the FVC2004 databases show improvements of this approach.
\end{abstract}

Keywords: Biometrics, fingerprint Sensor, Sensor Interoperability, Gabor filter.

\section{INTRODUCTION}

Biometric means identity verification of persons according to their physical or behavioral characteristics. Many physical body parts and personal features have been used for biometric systems: fingers, hands, irises, faces, ears, voices, gaits, odors, feet, signatures, and DNA ${ }^{1,2}$. A biometric system contains mainly a sensor module, a feature extraction module and a pattern matching module. A Sensor module acquires the raw biometric data of a person. Feature extraction module improves the quality of the captured image. Database module stores the biometric template information of enrolled Persons. Pattern matching module compares the present input with the stored template, which in-turn generates match score ${ }^{1}$.

Fingerprint-based identification is one of the most important biometric technologies. Humans have used fingerprints for personal identification and the validity of fingerprint identification has been well established. Segmentation is an important preprocessing step in fingerprint recognition system. The segmentation is partitioning the fingerprint image into multiple segments. Gabor functions play an important role in biometric recognition. The most prominent two steps are ${ }^{3}$ : 


\section{Foreground Segmentation}

The Gabor response is only computed in the foreground and, hence, foreground segmentation is required. Foreground region corresponds to the area containing ridges and valleys.

\section{Pixel-based Gabor response analysis}

Thresholds are deûned for the Gabor responses to associate a quality with each block. Similarly, thresholds are deûned to classify the pixel into good and poor quality depending on the neighboring region ${ }^{4,5}$.

They are employed for many physical or behavioral traits including iris, face, speech, gait, handwriting, palm print and ûngerprint recognition. Fingerprint recognition Gabor ûlterbank are used for the segmentation ${ }^{6}$ and improve the quality of databases and evaluate the performances of ûngerprint sensors and classiûcation and ûngerprint matching based on Gabor features. The Gabor filter is used for ûngerprint image enhancement ${ }^{7}$.

\section{Gabor-based Quality Classiûcation}

The Gabor-based quality classiûcation aids in classifying images into good and poor quality images ${ }^{8}$ :

\section{Good image}

clear ridges/valley contrast, easilydetected ridges, easily segmented.

\section{Poor image}

The poor quality images are also classiûed into dry (broken ridges, tiny parts of ridges can

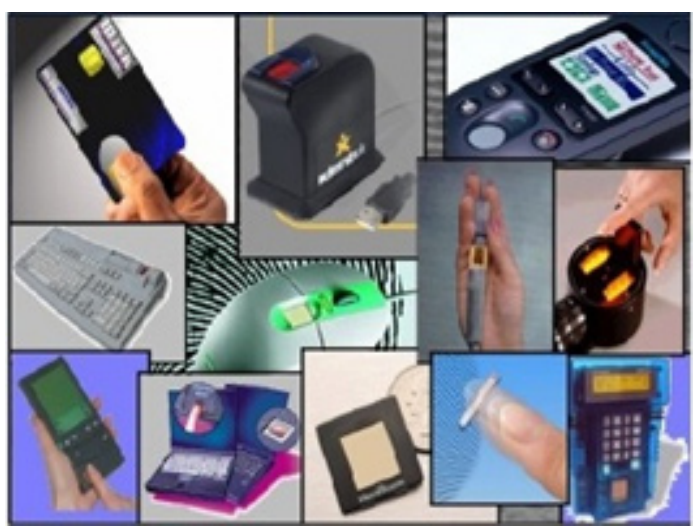

Fig. 1: comes in fingerprint section be separated) and smudged (not well separated ridges) on basis of the nature of the prints.

\section{Fingerprint Sensors}

Fingerprint sensors come in various shapes and sizes, but generally into two categories ${ }^{9}$ :

- Touch sensor: The user hold the finger on the sensor surface

- Swipe sensor: The user slides a finger vertically over the sensor surface.

There is the various acquisition technologies used in fingerprint sensors: optical, capacitive, thermal, and ultrasonic ${ }^{10,11}$.

\section{Optical sensor}

It capturing the digital image formed by the reflection of light from the points where ridges touches the sensors touch surface. Optical finger impression followers contain of a light sensor, touch surface and a capture device which can be a Charge Coupled Device.

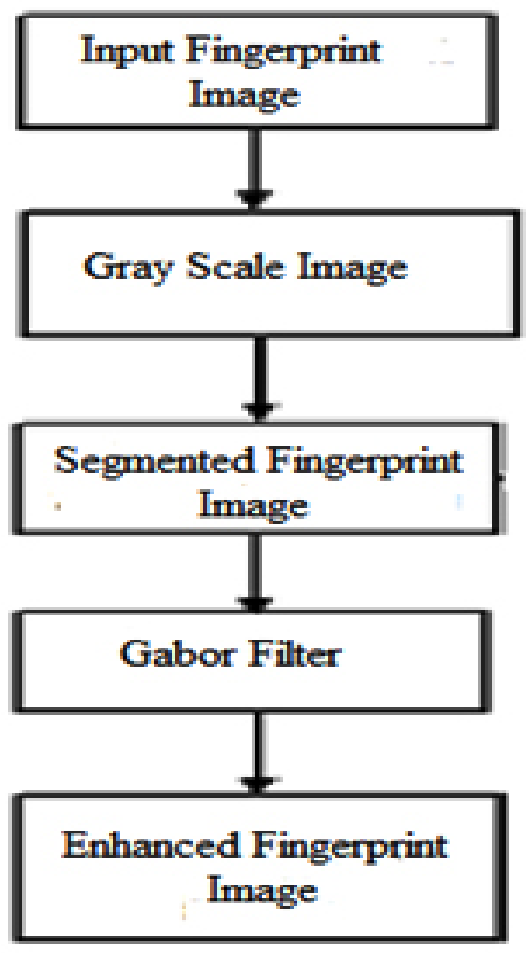

Fig. 2: Architecture of proposed scheme 


\section{Capacitive Sensor}

Capacitive sensor is the solid state sensor. Fingerprint sensors consist of an array of capacitive plates on a silicon chip. One plate of capacitor is formed by the finger; other plate holds a tiny area of metallization on the chip.

\section{Ultrasonic Sensor}

It uses high frequency sound waves to monitor the finger surface. Ultrasonic method of acquiring fingerprint is based on sending ultrasonic signals towards the finger and finds the echo.

\section{Thermal sensor}

Fingerprint sensors are made from the silicon die tiled by pixels of pyro-electric material that is sensitive to detect temperature differences. This sensor scans the surface of the finger, measuring the heat transferred from sensor to fingerprint.

\section{Sensor Interoperability}

Sensor interoperability is the ability of a biometric framework to adapt to the raw data obtained from a variety of sensors. Practically biometric frameworks would intended with look at information originated starting with the same sensor, but fail to give a good performance when the acquisition device is changed between the enrolment and the authentication phase [12]. Fingerprint sensor interoperability is the process of matching fingerprints collected from different sensors.

\section{Related Work}

Lugini et al. statistically analyzed how match scores change across different optical devices ${ }^{14}$.

Table 1: FVC 2004 Fingerprint Database

\begin{tabular}{|c|c|c|c|c|}
\hline $\begin{array}{l}\text { Data } \\
\text { base }\end{array}$ & $\begin{array}{c}\text { Sensor } \\
\text { Type }\end{array}$ & $\begin{array}{l}\text { Sensor } \\
\text { Name }\end{array}$ & $\begin{array}{c}\text { Image } \\
\text { Size }\end{array}$ & Res. \\
\hline DB1 & Optical & $\begin{array}{c}\text { V300 } \\
\text { Crossmatch }\end{array}$ & $\begin{array}{c}640 \times 480 \\
(307 \mathrm{~K} \\
\text { Pixels) }\end{array}$ & 500dpi \\
\hline DB2 & Optical & $\begin{array}{c}\text { Digital } \\
\text { Persona } \\
\text { U.are.U4000 }\end{array}$ & $\begin{array}{c}328 \times 364 \\
(119 \mathrm{~K} \\
\text { pixels })\end{array}$ & 500dpi \\
\hline DB3 & Thermal & $\begin{array}{c}\text { Atmel } \\
\text { FCD4B } \\
14 \mathrm{CB}\end{array}$ & $\begin{array}{c}300 \times 480 \\
(144 \mathrm{~K} \\
\text { pixels })\end{array}$ & 512dpi \\
\hline
\end{tabular}

Results of the Kendall's rank correspondence test pointed out that there is a significant difference

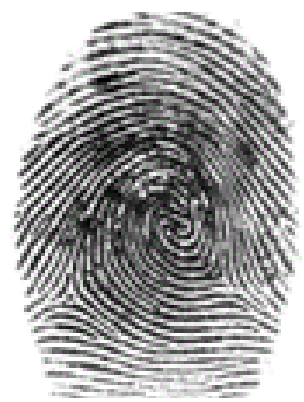

Fig. 3: Original fingerprint image taken from DB1

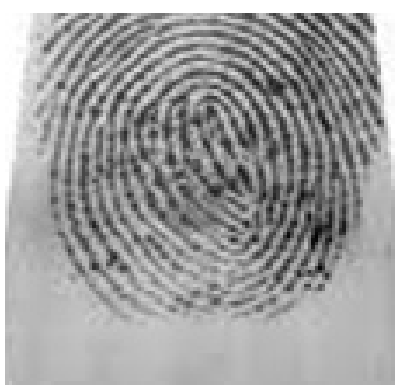

Fig. 4: Original fingerprint image taken from DB2

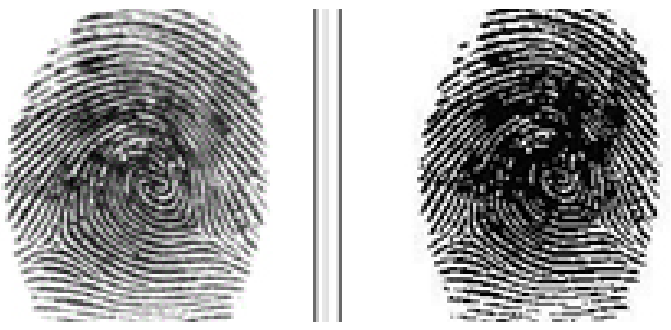

Fig. 5:Original fingerprint of good quality taken from DB1 and Segmented image of good quality

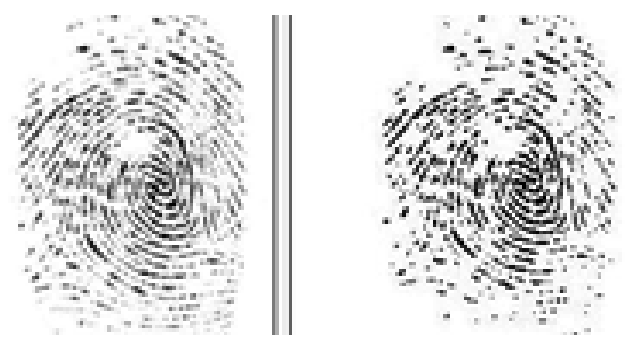

Fig. 6: Original fingerprint of Poor quality taken from DB1 and Segmented image of poor quality 

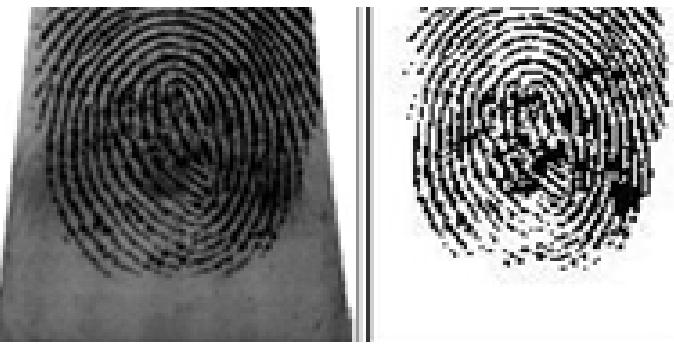

Fig. 7: Original fingerprint of good quality image taken from DB2 and Segmented image of good quality
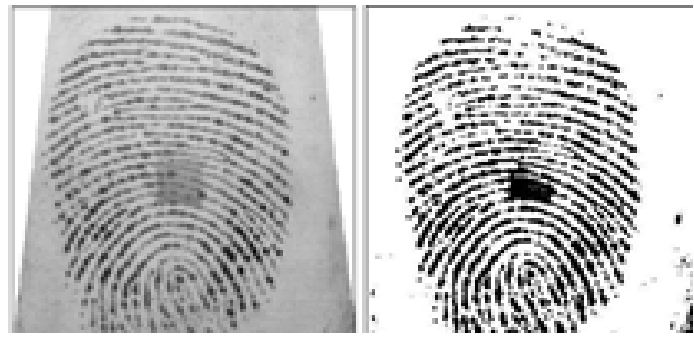

Fig. 8: Original fingerprint of poor quality image taken from DB2 and Segmented image of poor quality
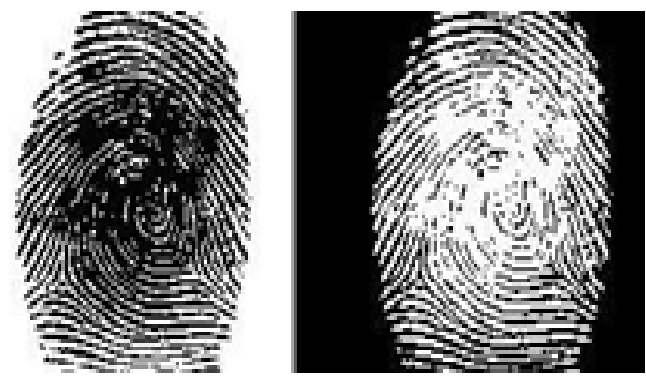

Fig. 9: Segmented fingerprint of good quality image taken from DB1 and Gabor filter image of good quality
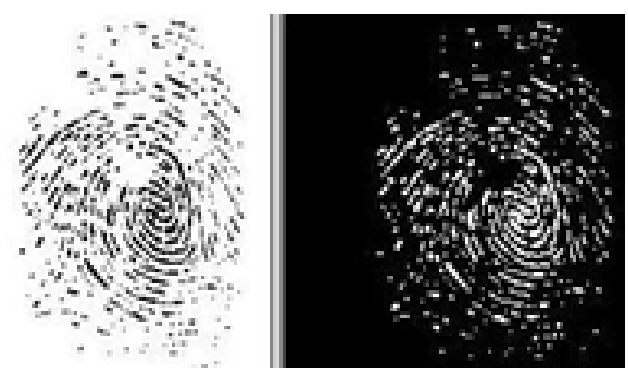

Fig. 10: Segmented fingerprint of Poor quality image taken from DB1 and Gabor filter image of poor quality between sensor pairs and that those change will not symmetric when inverting those two devices.

Marasco et al. proposed a learning-based approach to improve cross-device performance ${ }^{15}$. They extracted quality and intensity-based characteristics of fingerprint images acquired using four different commercial optical de-vices
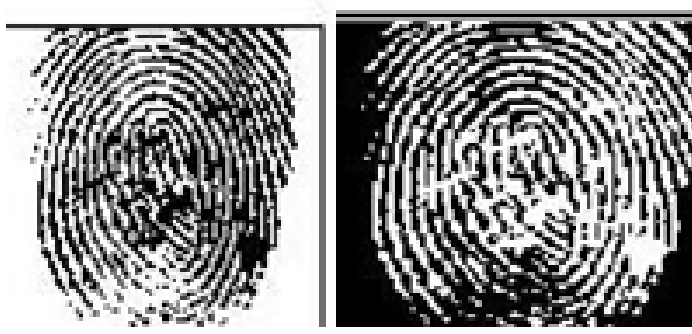

Fig. 11: Segmented fingerprint of good quality image taken from DB2 and Gabor filter image of good quality
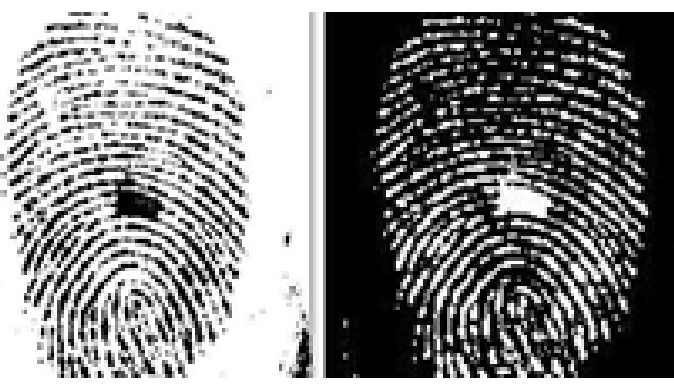

Fig. 12: Segmented fingerprint of poor quality image taken from DB2 and Gabor filter image of poor quality

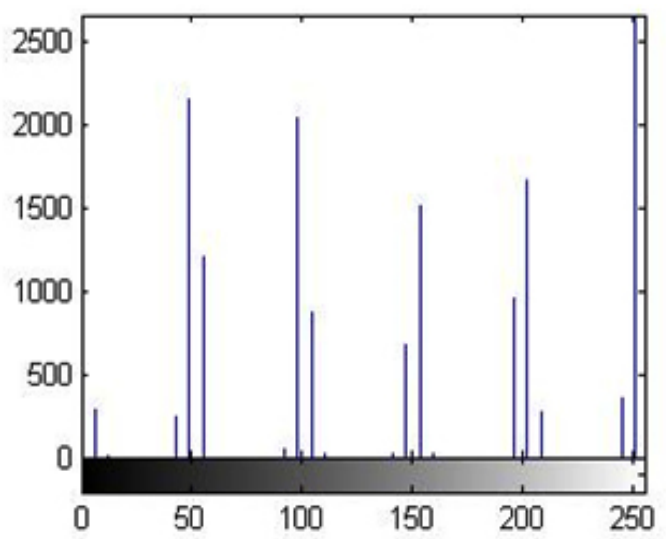

Fig. 13: Histogram of original image of good quality taken from DB1 


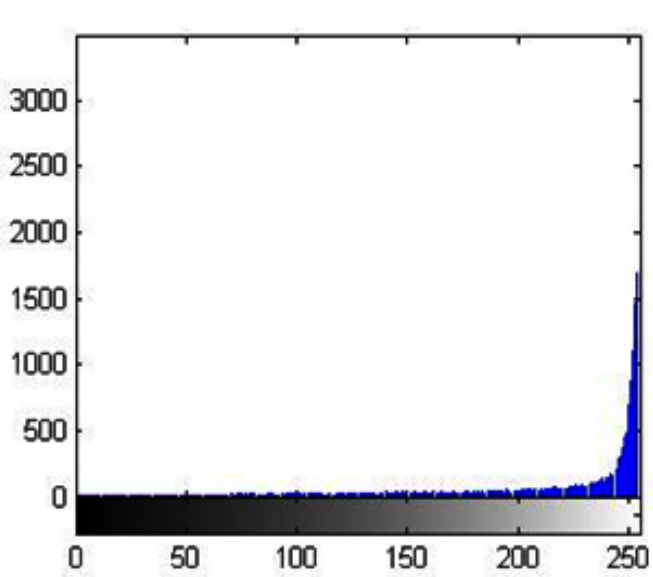

Fig. 14: Histogram of original image of poor quality taken from DB1

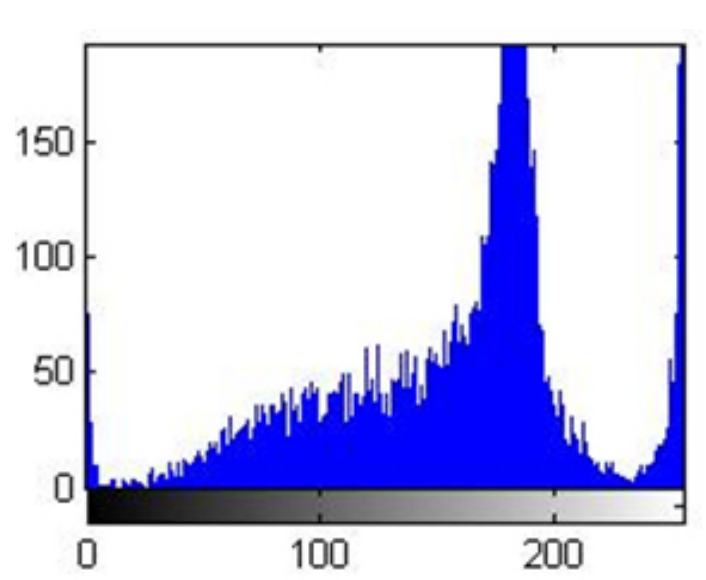

Fig. 15: Histogram of original image of good quality taken from DB2

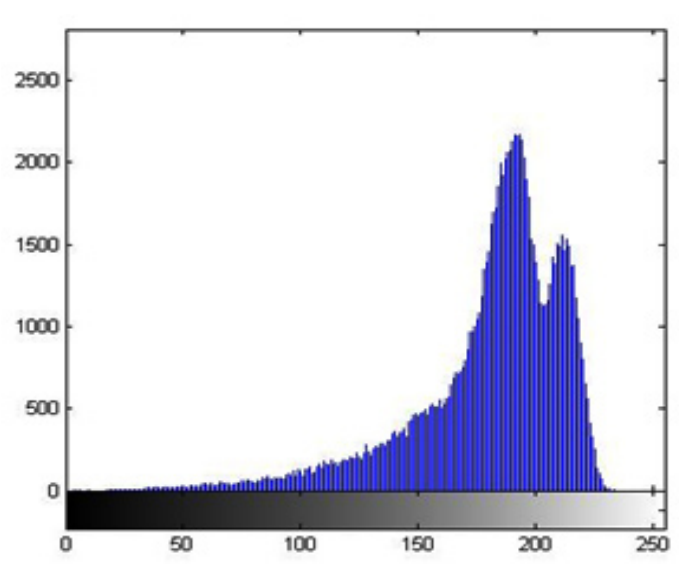

Fig. 16: Histogram of original image of poor quality taken from DB2

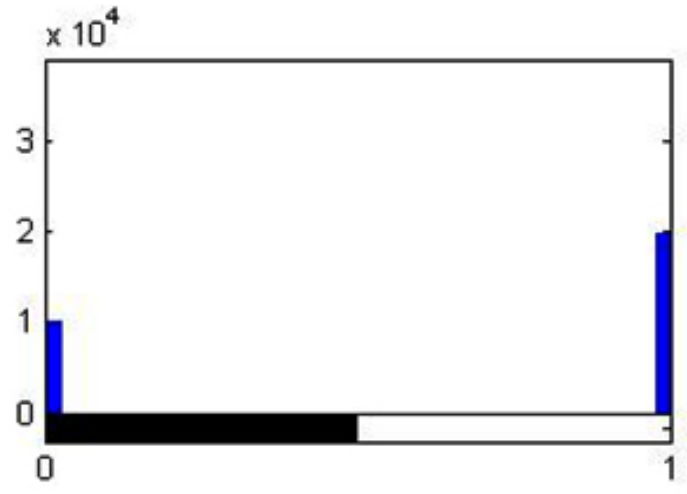

Fig. 17: Histogram of segmented image of good quality taken from DB1

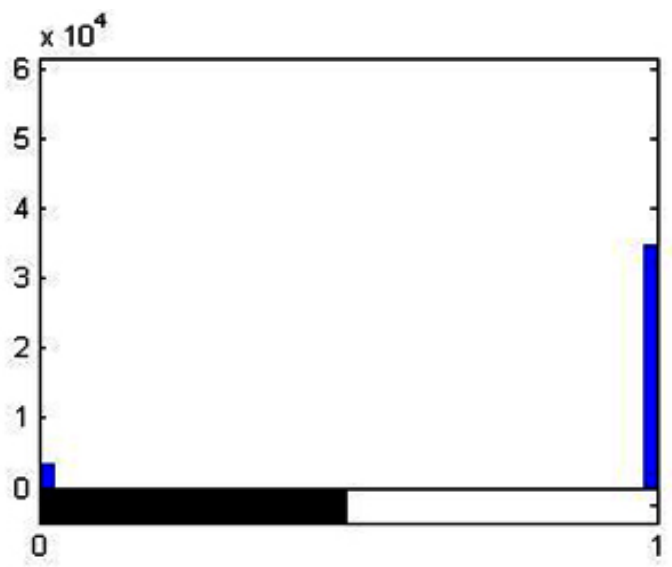

Fig. 18: Histogram of segmented image of poor quality taken from DB1

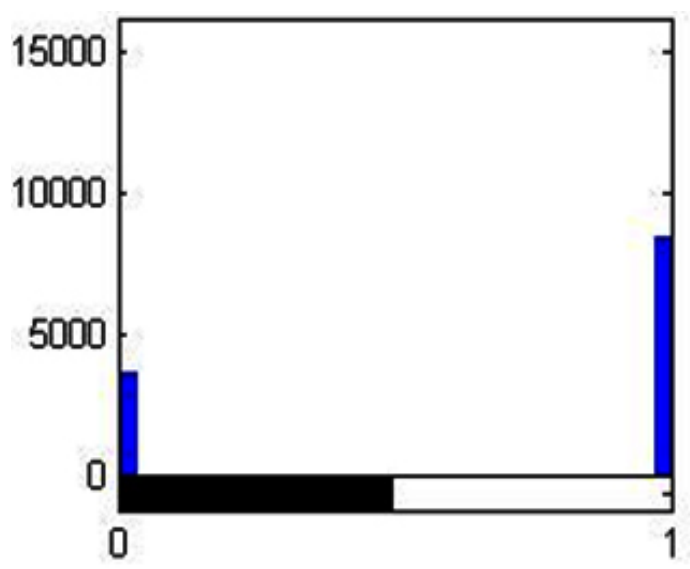

Fig. 19: Histogram of segmented image of good quality taken from DB2 


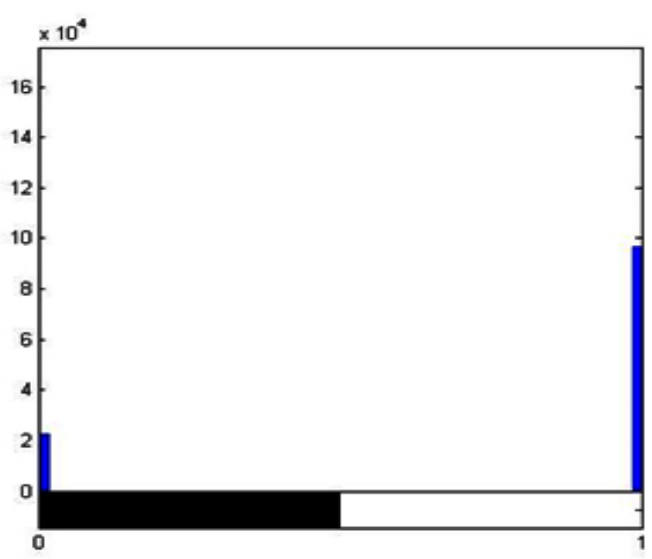

Fig. 20: Histogram of segmented image of poor quality taken from DB2

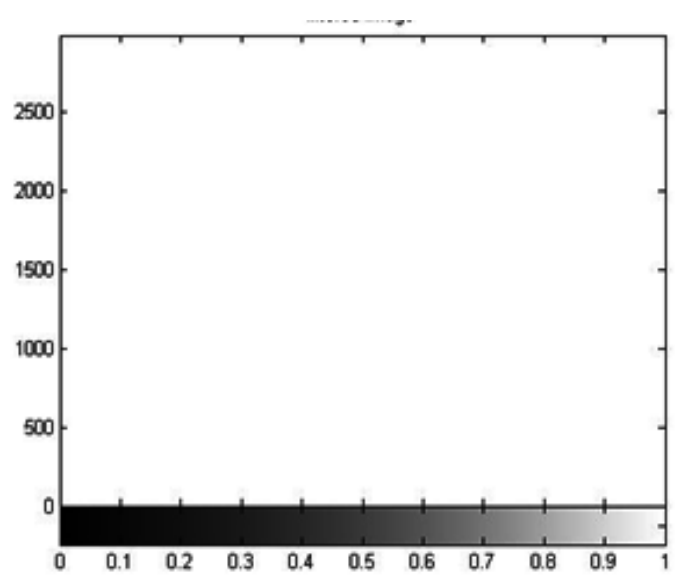

Fig. 21: Histogram of Gabor filter image of good quality taken from DB1

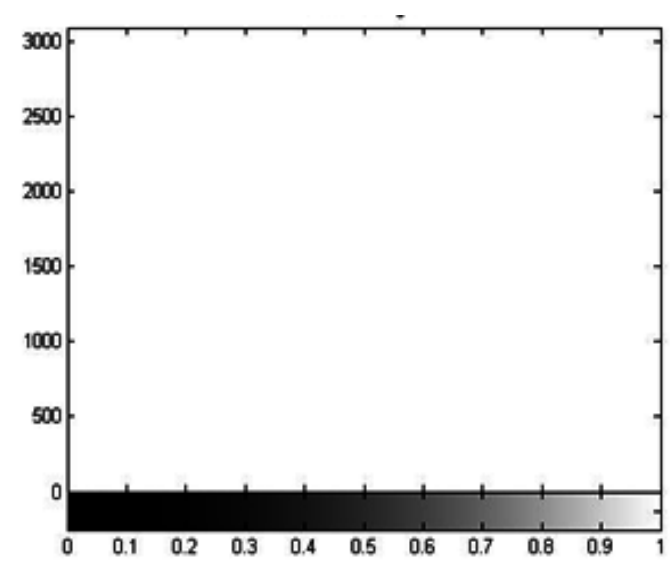

Fig. 22: Histogram of Gabor filter image of poor quality taken from DB1

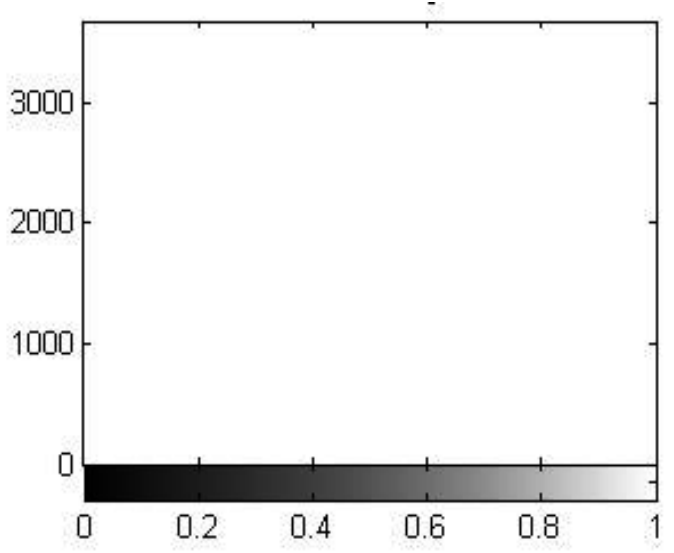

Fig. 23: Histogram of Gabor filter image of good quality taken from DB2

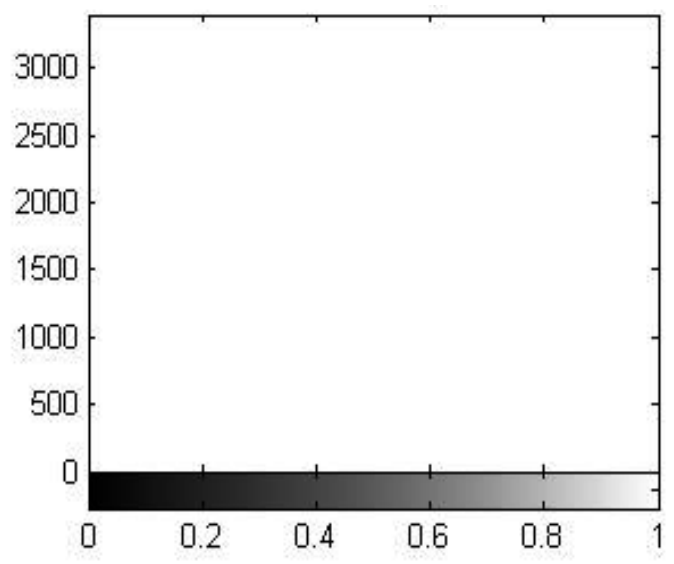

Fig. 24: Histogram of Gabor filter image of poor quality taken from DB2

and scanned ink rolled prints. The defined set of characteristics was concatenated with the match score into a feature vector used for training a pattern classifier. Later, they investigated the impact of age and gender across different optical devices and matching algorithms ${ }^{16}$.

Satish Kumar Chavan, Parth Mundada, Devendra Pal discussed fingerprint authentication using Gabor filter which is used to extract features of fingerprint images ${ }^{17}$. FVC 2000 and DBIT fingerprint databases are used to evaluate the algorithm and it achieves the average efficiency of $82.95 \%$ and $89.68 \%$ respectively.

Lu Yang, Gongping Yang, Lizhen Zhou, Yilong Yin proposed a super pixel based finger vein region of 
interest (ROI) extraction with sensor interoperability in biometric systems ${ }^{19}$. Finger boundaries were firstly determined by tracking super pixels. Then the middle points of detected finger boundaries were used to adjust the finger directions. Finally, internal tangents of finger boundaries were used to localize ROI. It was found that this method extracts $\mathrm{ROI}$ accurately from the images acquired using multiple sensors.

\section{Proposed Framework}

A fingerprint sensor is to obtain a good quality image of the ridge pattern. The quality of a fingerprint image depends on sensor characteristics and the condition of the finger surface (i.e. good, dry). Sensing mechanism of each device is different and images with different sizes, resolution, feature distribution, gray level are produced.

In this work, a Novel approach to address sensor interoperability using Gabor filter is presented. This approach avoids the requirement to change the model when the sensing devices changes. The approach follows procedures that enhances segmentation process of quality images and apply on Gabor filter. Gabor filter is used for ûngerprint image enhancement. The fingerprint image enhancement is to improving the clarity of the ridge and valley structure.

\section{Database}

The database used is an FVC2004 database. The analysis for this study was conducted using fingerprints collected from three different fingerprint sensors. The three fingerprint sensors are V300 Crossmatch, Digital Persona U.are. U4000, and Atmel Finger Chip FCD4B14CB. The V300 Crossmatch and Digital Persona U.are. U4000 sensors are optical sensors and Finger Chip FCD4B14CB is a thermal sensor. Table 1 shows the database, sensors and specifications for the three fingerprint sensors:

\section{RESULTS}

In this proposed framework there are 2 fingerprint images of different optical sensors one is V300 Crossmatch and other is of Digital Persona U.are.U4000. This approach follows a procedure that enhances and segments the quality image then applies on Gabor filter.

\section{Gray-Scale \\ Segmentation}

Fingerprint segmentation technique can improve the accuracy in collecting clear fingerprint area and mark noise areas. The segmented images of good and poor quality fingerprint are illustrated in figure below:

\section{Gabor Filter}

Gabor filter is used for enhance the segmented fingerprint image for better result. The images are illustrated in figures below:

\section{Histogram}

Histograms of original image, segmented image and Gabor filter Image of good and poor quality were constructed. It is found that histograms of the Gabor filter images are similar and intensities lie in the range 0-1 as shown below:

\section{CONCLUSION}

In this work, the proposed technique improves sensor interoperability in fingerprint sensors by using a Gabor filter. The Proposed approach is used for enhance the segmented fingerprint image of good quality and poor quality. It improves sensor interoperability in fingerprint sensors. In this paper, a Gabor Filter approach is presented to enhance the segmented images accurately when the image is captured using various sensors. In future, it will applicable on any type of biometric sensor.

\section{REFERENCE}

1. RRaghavendra, Rao Ashok, and G Hemantha Kumar, "Multimodal biometric score fusion using gaussian mixture model and monte carlo method", Journal of Computer Science and Technology, 25(4):771-782, 2010.

2. Renu Bhatia, "Biometrics and face recognition 
techniques," International Journal of Advanced Research in Computer Science and Software Engineering (IJARCSSE), vol. 3, issue5, pp. 93-99, May 2013.

3. Arun Ross, Chair Lawrence Hornak, Xin Li, "Facilitating Sensor Interoperability and Incorporating Quality in Fingerprint Matching Systems", Lane Department of Computer Science and Electrical Engineering, November 2014

4. L. Shen, A. Kot, and W. Koo, "Quality measure of ûngerprint images," in Audio - and Videobased Biometric Person Authentication (AVBPA), 2001, pp. 266-271.

5. G. Vallarino, G. Gianarelli, J. Barattini, A. Gomez, A. Fernandez, and A. Pardo, "Performance improvement in a ûngerprint classiûcation system using anisotropic diûusion," CIARP - Iberoamerican Congress of Pattern Recognition, vol. LNCS 3287, pp. 582-588, 2004.

6. F. Alonso-Fernandez, J. Fierrez-Aguilar and J. Ortega-Garcia, "An enhanced Gabor ûlterbased segmentation algorithm for ûngerprint recognition systems", Proc. 4th Int. Symposium on Image and Signal Processing and Analysis (ISPA2), Zagreb, Croatia, pp. 239-244, 2005.

7. Carsten Gottschlich, "Curved Gabor Filters for Fingerprint Image Enhancement”, arXiv: 1104.4298v2 [cs.CV] 25 Jul 2014.

8. Chaohong Wu, Sergey Tulyakov and Venu Govindaraju, "Image Quality Measures for Fingerprint Image Enhancement", Center for Uniûed Biometrics and Sensors (CUBS) SUNY at Buffalo, USA.

9. Shahzad Memon, Mojtaba Sepasian, Wamadeva Balachandran, "Review of Fingerprint Sensing Technologies", Conference Paper January 2009.

10. Salil Prabhakar, Alexander Ivanisov, and Anil Jain, "Biometric recognition: sensor characteristics and image quality," IEEE Instrumentation \& Measurement Magazine, pp. 1094-6969, June 2011.
11. Davide Maltoni, Dario Maio, Anil Jain, Salil, Handbook of Fingerprint Recognition, Chapter 2.

12. A. Ross, K. Nandakumar, and A.K. Jain, Handbook of multibiometrics, Springer, 2006.

13. Emanuela Marasco, Zachary Chapman, Bojan Cukic, "Improving Fingerprint Interoperability by Integrating Wavelet Entropy and Binarized Statistical Image Features", 2016.

14. Lugini, L.; Marasco, E.; Cukic, B.; Gashi, I.: Interoperability in Fingerprint Recognition: a Large-Scale Study. Workshop on Reliability and Security Data Analysis (RSDA), Budapest, pp. 1-6, June 2013.

15. Marasco, E.; Lugini, L.; Cukic, B.: Minimizing the Impact of Low Interoperability between Optical Fingerprint Sensors. Biometrics: Theory, Applications and Systems (BTAS), pp. 1-8, 2013.

16. Marasco, E.; Lugini, L.; Cukic, B.: Automatic Enhancement of Interoperability between Optical Fingerprint Sensors. NIST International Biometric Performance Testing Conference (IBPC), 2014.

17. Satish kumar Chavan, Parth Mundada, Devendra Pal, "Fingerprint Authentication using Gabor Filter based Matching Algorithm", 2015 International Conference on Technologies for Sustainable Development (ICTSD2015), Feb. 04 - 06, 2015, Mumbai, India.

18. Sunpreet Singh Arora, "fingerprint recognition: contributions to latent matching and $3 d$ fingerprint target generation", Michigan State University in partial fulûllment of the requirements for the degree of Computer Science - Doctor of Philosophy 2016.

19. Lu Yang, Gongping Yang, Lizhen Zhou, Yilong Yin, "Super pixel based finger vein roi extraction with sensor interoperability," IEEE International Conference on Advances in Biometric (ICB) 2015, pp. 444-451, May 19-22, 2015. 